\title{
Strain-engineered direct-indirect band gap transition and its mechanism in two-dimensional phosphorene
}

\author{
Xihong Peng, ${ }^{1, *}$ Qun Wei, ${ }^{1,2}$ and Andrew Copple ${ }^{3}$ \\ ${ }^{1}$ School of Letters and Sciences, Arizona State University, Mesa, Arizona 85212, USA \\ ${ }^{2}$ School of Physics and Optoelectronic Engineering, Xidian University, Xi' an, 710071, P.R. China \\ ${ }^{3}$ Department of Physics, Arizona State University, Tempe, Arizona 85287, USA \\ (Received 18 May 2014; revised manuscript received 16 July 2014; published 4 August 2014)
}

\begin{abstract}
Recently fabricated two-dimensional phosphorene crystal structures have demonstrated great potential in applications of electronics. In this paper, strain effect on the electronic band structure of phosphorene was studied using first-principles methods including density functional theory (DFT) and hybrid functionals. It was found that phosphorene can withstand a tensile stress and strain up to $10 \mathrm{~N} / \mathrm{m}$ and $30 \%$, respectively. The band gap of phosphorene experiences a direct-indirect-direct transition when axial strain is applied. A moderate $-2 \%$ compression in the zigzag direction can trigger this gap transition. With sufficient expansion $(+11.3 \%)$ or compression ( $-10.2 \%$ strains), the gap can be tuned from indirect to direct again. Five strain zones with distinct electronic band structure were identified, and the critical strains for the zone boundaries were determined. Although the DFT method is known to underestimate band gap of semiconductors, it was proven to correctly predict the strain effect on the electronic properties with validation from a hybrid functional method in this work. The origin of the gap transition was revealed, and a general mechanism was developed to explain energy shifts with strain according to the bond nature of near-band-edge electronic orbitals. Effective masses of carriers in the armchair direction are an order of magnitude smaller than that of the zigzag axis, indicating that the armchair direction is favored for carrier transport. In addition, the effective masses can be dramatically tuned by strain, in which its sharp jump/drop occurs at the zone boundaries of the direct-indirect gap transition.
\end{abstract}

DOI: 10.1103/PhysRevB.90.085402

PACS number(s): 73.22.-f, 62.20.D-, 62.23.Kn

\section{INTRODUCTION}

Two-dimensional (2D) layered crystal materials have attracted extensive research efforts in recent years, such as graphene [1,2] and molybdenum disulfide [3], for their potential applications in future electronics. Most recently, researchers have successfully fabricated new 2D few-layer black phosphorus [4-7] and found that this material is chemically inert and has great transport properties. It was reported that it has a carrier mobility up to $1000 \mathrm{~cm}^{2} / \mathrm{V} \cdot \mathrm{s}^{4}$ and an on/off ratio up to $10^{4}$ [5] was achieved for the phosphorene transistors at room temperature. In addition, this material shows a finite direct band gap at the center of Brillouin zone [4,5,8-10] (in contrast to the vanishing gap in graphene), which creates the potential for additional applications in optoelectronics.

Tailoring electronic properties of semiconductor nanostructures has been critical for their applications. Strain has long been used to tune electronic properties of semiconductors $[11,12]$. As a practical issue, strain is almost inevitable in fabricated monolayer nanostructures, manifesting as the formation of ridges and buckling [13,14]. A more interesting case comes from intentionally introduced and controlled strains, though. Methods for introducing strain include lattice mismatch, functional wrapping $[15,16]$, material doping $[17,18]$, and direct mechanical application [19]. It was found that nanostructures maintain integrity under a much higher strain than their bulk counterparts [20,21], which dramatically expands the strength of applicable strain to nanostructures. In particular, 2D layered materials, such as graphene and $\mathrm{MoS}_{2}$, possess superior mechanical flexibility and can sustain

\footnotetext{
*xihong.peng@asu.edu
}

a spectacularly large strain $(\geq 25 \%)$ [22-24]. We calculated the stress-strain relation in phosphorene and found that it can withstand a tensile strain up to $30 \%$. Compared to other 2D materials such as graphene, phosphorene demonstrates superior flexibility with an order of magnitude smaller Young's modulus [25]. This is especially useful in practical large magnitude-strain engineering of this material.

As already demonstrated by several research groups, strain shows remarkable effects in modifying the electronic properties of phosphorene. For example, Rodin et al. [8], using density functional theory (DFT) and tight-binding models, predicted an anisotropic dispersion relation with a direct band gap for phosphorene. They analyzed the localized orbital composition of the band edge states and suggested a semiconductor-to-metal transition with compression. Liu et al. [5] briefly reported the sensitive dependence of the band gap on in-layer stress and a critical compressive strain of $3 \%$ to trigger the direct-to-indirect band gap transition. Fei and Yang [26] theoretically predicted that the preferred conducting direction in phosphorene can be rotated by $90^{\circ}$ with an appropriate biaxial or uniaxial strain based on the anisotropicity of the material $[5,8,27]$.

However, a full picture of detailed and systematic analysis of the strain effect on the band structure is still missing. For example, what is the elastic limit for phosphorene? Where is the conduction/valence band edge located when the band gap becomes indirect? Is there any additional critical strain to trigger the band gap transition? What is the mechanism/origination for this direct-indirect band gap transition? In this paper, we will answer these questions by providing tension-strain relation and a full analysis of strain effects on the band structure of phosphorene. By revealing the evolution of band structure with strain, it is clear to see the shift of the band edges and 
gap transitions. Several critical strains have been identified to trigger the direct-indirect transition. In addition, with sufficient large tensile $(+11.3 \%)$ or compressive $(-10.2 \%)$ strain, the indirect band gap was found to become direct again. Five strain zones with distinct band structure were identified. This detailed analysis on the direct-indirect band gap transition is crucial for optical applications of phosphorene. For example, Peng and Copple at Arizona State University predicted a similar direct-indirect band gap transition in GaAs nanowires with uniaxial strains $[28,29]$ and the gap transition was observed in a recent experiment [30] in which the luminescence of GaAs nanowires can be switched on and off under the influence of a uniaxial stress.

In this paper, we also discuss in detail the mechanism for the gap transition by examining the bond nature of near-bandedge electronic orbitals. This mechanism has been applied successfully in many other semiconductor nanostructures [28,29,31-38]. Effective masses of charge carriers (thus carrier mobility) were also found to be drastically tuned by strain.

\section{SIMULATION DETAILS}

The first-principles DFT [39] calculations were carried out using the Perdew-Burke-Ernzerhof (PBE) exchangecorrelation functional [40] along with the projector-augmented wave potentials [41,42] for the self-consistent total energy calculations and geometry optimization. Both standard DFT with generalized gradient approximation (GGA) and hybrid functional $[43,44]$ methods were adopted to investigate the strain effect on the electronic properties of phosphorene. In the hybrid Heyd-Scuseria-Ernzerhof (HSE)06 method [43,44], the exchange-correlation functional uses a mixing parameter to incorporate Hartree-Fock (HF) exact exchange functional and the PBE functional. In this study, the fraction of the HF exchange was set to be the default value $\alpha=0.25$. Both methods of the DFT-PBE and HSE06 give consistent results of the strain effects on the electronic band structures, including the direct-indirect band gap transition and the gap variation trends with strain.

The calculations were performed using the Vienna $A b$ initio Simulation Package $[45,46]$. The kinetic energy cutoff for the plane wave basis set was chosen to be $350 \mathrm{eV}$. The reciprocal space was meshed at $14 \times 10 \times 1$ using the Monkhorst-Pack method. The energy convergence criteria for electronic and ionic iterations were set to be $10^{-5} \mathrm{eV}$ and $10^{-4} \mathrm{eV}$, respectively. Such parameter settings ensure the calculations were converged within $5 \mathrm{meV}$ in total energy per atom. To simulate a monolayer of phosphorene, a unit cell with periodic boundary condition was used. A vacuum space of at least $16 \AA$ was applied to minimize the interaction between layers. In band structure calculations, 21 points were collected along each high symmetry line in reciprocal space.

Unlike a flat structure of graphene, the single layer black phosphorus has a puckered honeycomb structure with each phosphorus atom covalently bonded with three adjacent atoms (see Fig. 1). The initial structure of phosphorene was obtained from black phosphorus [47]. Our calculated lattice constants for bulk black phosphorus are $a=3.308 \AA, b=4.536 \AA$, and $c=11.099 \AA$, in good agreement with experimental values [47] and other theoretical calculations [5,27]. The relaxed lattice

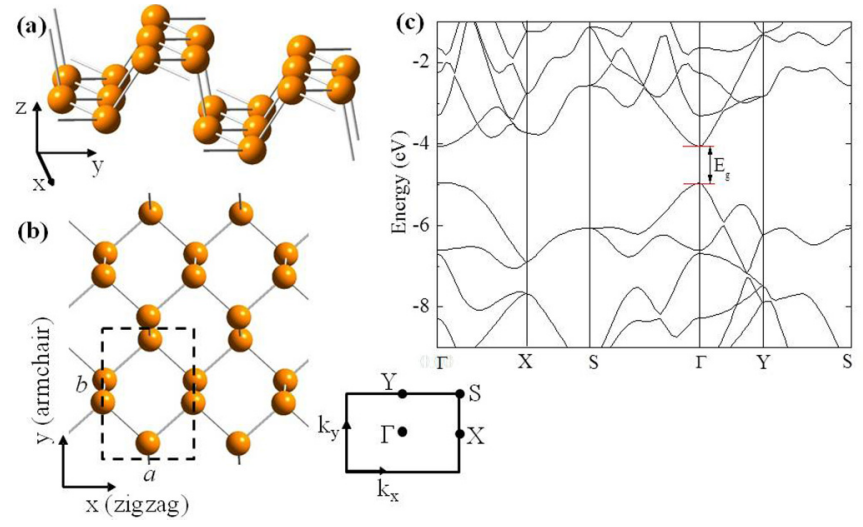

FIG. 1. (Color online) Snapshots of 2D phosphorene and its band structure. The dashed rectangle in (b) indicates a unit cell. The energy in the band structure is referenced to vacuum level.

constants for a monolayer of phosphorene are $a=3.298 \AA$ and $b=4.627 \AA$.

Starting with the relaxed phosphorene, strain within the range of $\pm 12 \%$ was applied in either the $x$ (zigzag) or $y$ (armchair) direction to explore its effects on the band structure. The applied strain is defined as $\varepsilon_{x}=\frac{a_{x}-a_{x 0}}{a_{x 0}}, \varepsilon_{y}=\frac{a_{y}-a_{y 0}}{a_{y 0}}$, where $a_{x}\left(a_{y}\right)$ and $a_{x 0}\left(a_{y 0}\right)$ are the lattice constants along the $x(y)$ direction for the strained and relaxed structures, respectively. The positive values of strain refer to expansion, while negative corresponds to compression. With each axial strain applied, the lattice constant in the transverse direction was fully relaxed through the technique of energy minimization to ensure the force in the transverse direction is a minimum.

\section{RESULTS AND DISCUSSIONS}

\section{A. Stress-strain relation and critical strain limit of phosphorene}

Generally, a compression applied in an axial direction results in an expansion in the transverse direction. The applied axial strains in the $x(y)$ direction and the transverse strain response in the $y(x)$ direction are reported in Fig. 2(a). The Poisson's ratio, $v=-\frac{d \varepsilon_{\text {transverse }}}{d \varepsilon_{\text {axial. }}}$, was found to be 0.7 and 0.2 for the axial strain applied in the zigzag and armchair directions, respectively. These significantly different Poisson's ratios further indicate the anisotropic nature of phosphorene. Figure 2(b) presents the change in the total energy of phosphorene as a function of the applied axial strain. The deeper energy surface for $\varepsilon_{x}$ suggests that strain is more difficult to apply in the zigzag than the armchair direction.

To estimate the elastic limit of phosphorene, we calculated the stress (force per unit length) [48] of phosphorene as a function of tensile strain, using the method described in the references [49,50]. This method of calculating stressstrain relation was originally introduced for three-dimensional crystals. In a 2D monolayer phosphorene, the stress calculated from the Hellmann-Feynman theorem was modified to be the force per unit length [48]. The tensile strain is applied in either the zigzag or armchair direction. Our calculated stress-strain relation is presented in Fig. 2(c). It shows that phosphorene can sustain a stress up to $10 \mathrm{~N} / \mathrm{m}$ and $4 \mathrm{~N} / \mathrm{m}$ in the zigzag and armchair directions, respectively. The 

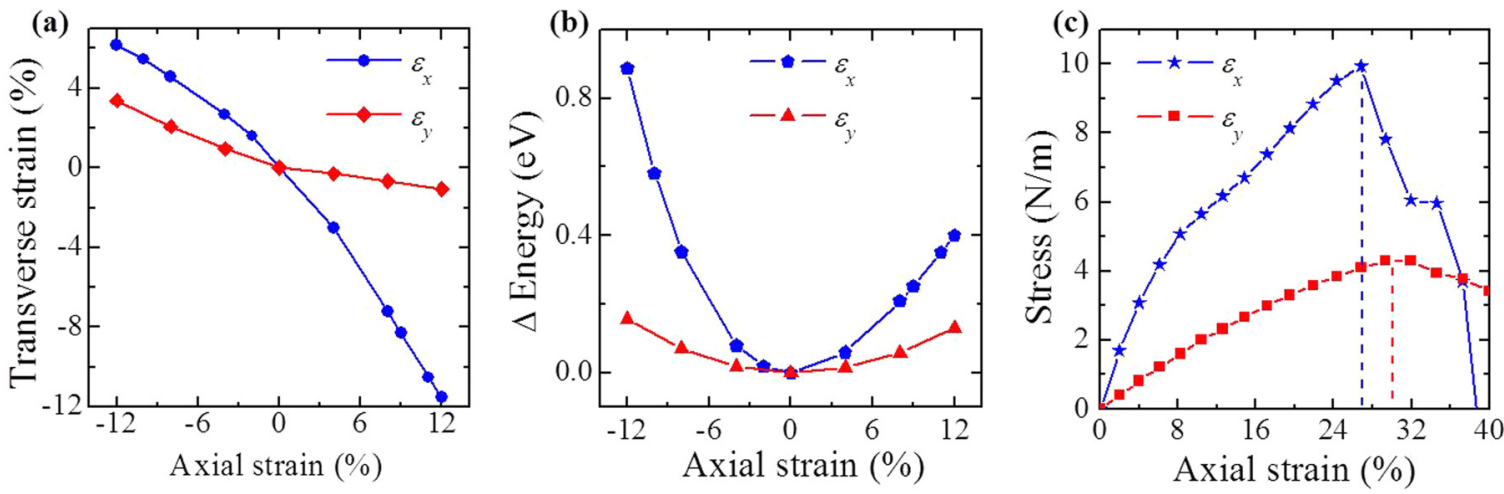

FIG. 2. (Color online) (a) The applied axial strains in the $x$ and $y$ axes and their transverse strain response in the $y$ and $x$ directions, respectively. (b) The change in the total energy as a function of the applied strain. (c) The stress as a function of tensile load for phosphorene. Phosphorene can withstand a critical tensile strain up to $30 \%$ in the armchair direction. The different behaviors along the $x$ and $y$ directions in (a)-(c) indicate the anisotropic nature of phosphorene.

corresponding tensile strain limits are $27 \%$ and $30 \%$ along the zigzag and armchair axes, respectively. This predicted elastic strain limit is close to that found in other 2D materials such as graphene and $\mathrm{MoS}_{2}$ [22-24], suggesting that phosphorene is highly flexible and may have potential applications in flexible displays.

\section{B. Strain effect on the band structure of phosphorene}

Our DFT predicted band gap for 2D phosphorene is $0.91 \mathrm{eV}$, which is in agreement with other theoretical work $[5,10]$. It is well known that DFT underestimates the band gap of semiconductors, and the advanced Green's function method (GW) can provide improved predictions. The $G W$ gap for phosphorene calculated by Tran et al. [10] is about $2.0 \mathrm{eV}$. However, the present work is mainly focused on the strain effect on the band structure, and the advanced hybrid functional method HSE06 gives results of the strain effects on the electronic band structures consistent with that of the DFT-PBE method. In addition, previous studies [32] on Si nanoclusters showed that the energy gap calculated by DFT obeys a similar strain-dependency as the optical gap predicted by the advanced configuration interaction method and the quasiparticle gap. Therefore, we expect that the DFT can correctly predict the general trends of strain effect on the band structure and near-gap states in phosphorene.

By comparing the band structure of phosphorene at different values of axial strains, we found that strain has a remarkable effect on the band structure along two particular $K$ directions, $\Gamma$ to $X(0.5,0,0)$ and $\Gamma$ to $Y(0,0.5,0)$, respectively. For simplification, we only plot the band structure along these two directions in Figs. 3 and 4.

Figure 3 presents the effect of strain $\varepsilon_{x}$ (applied in the zigzag direction) on the band structure. Figure 3(f) is for the relaxed phosphorene with a direct band gap at $\Gamma$. With an increase of tensile strain, the band gap becomes indirect, then transitions back to direct. For example, at $\varepsilon_{x}=+9 \%$, it shows an indirect band gap with the conduction band minimum (CBM) shifted from $\Gamma$ to $K 1(0,0.3,0)$, while the valence band maximum (VBM) remains at $\Gamma$. At $\varepsilon_{x}=+12 \%$, it gives a direct gap with both the CBM and VBM at $K 1(0,0.3,0)$. Similarly, with an increase in compression, the gap first transitions to indirect

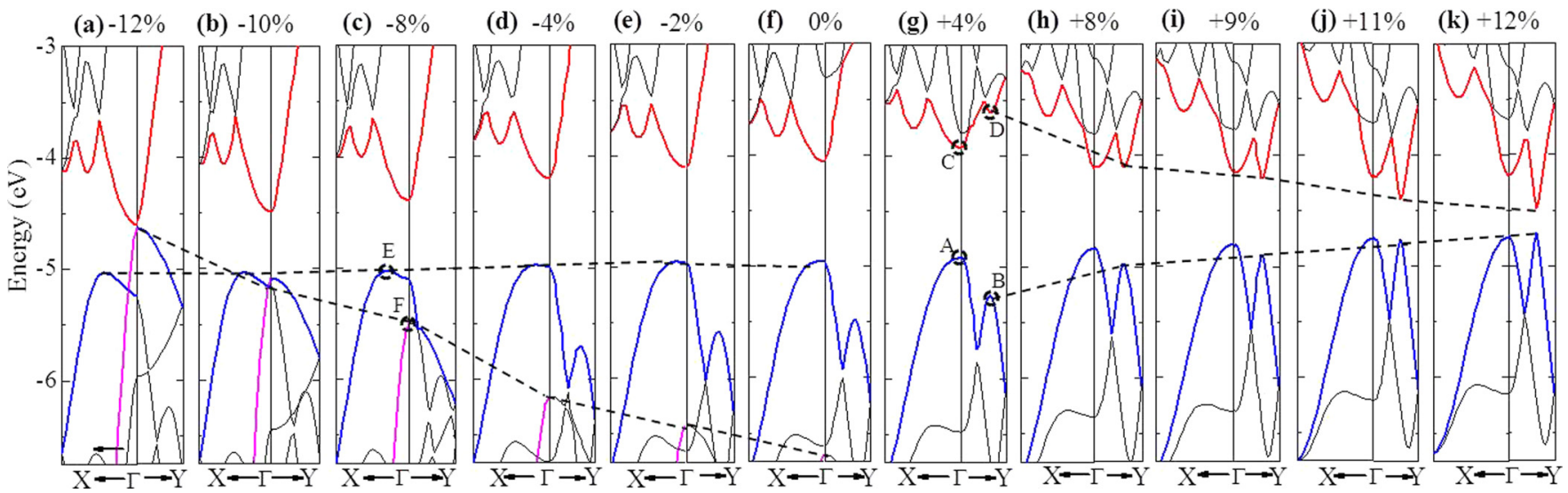

FIG. 3. (Color online) The strain $\varepsilon_{x}$ (applied in the zigzag direction) manipulated direct-indirect band gap transition in 2D phosphorene. Positive strain indicates expansion, while negative corresponds to compression. All energies are referenced to vacuum level. Starting from the relaxed structure, the band gap experiences a direct-indirect-direct transition with both tensile and compressive strain. The direct/indirect nature of the band gap is the result of the competition among the energies of near-band-edge states A-F. The dashed lines are guide for eye for the energy shifts of states B, D, E, and F. 


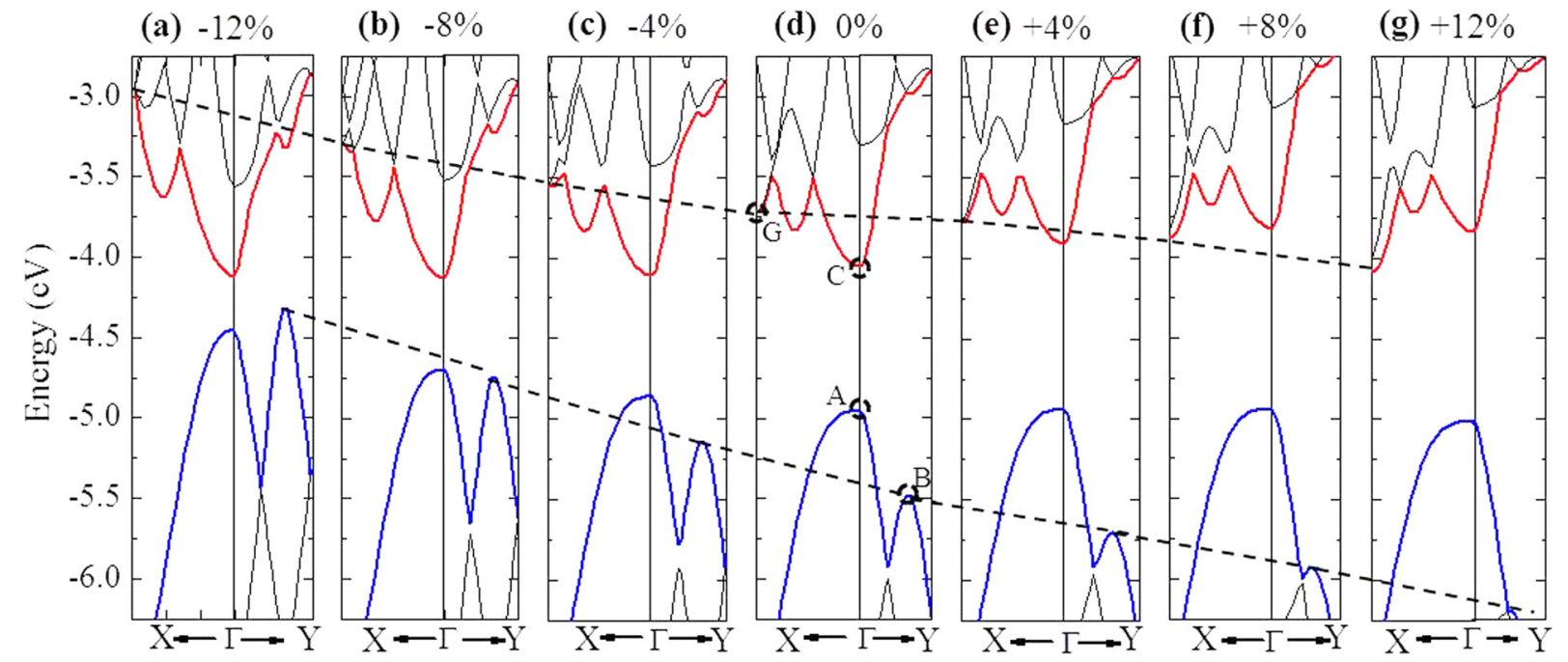

FIG. 4. (Color online) The strain $\varepsilon_{y}$ (applied in the armchair direction) manipulated direct-indirect band gap transition in phosphorene. All energies are referenced to vacuum level. The band gap shows a direct-indirect transition with expansion/compression. The direct/indirect nature of the gap results from the energy competition of near-band-edge states A, B, C, and G. The dashed lines are a guide for viewing the energy shifts of states B and G.

and then back to direct. For instance, at $\varepsilon_{x}=-8 \%$, the gap is indirect with the CBM remaining at $\Gamma$ while the VBM shifted to $K 2(0.15,0,0)$. When $\varepsilon_{x}=-12 \%$, it shows a direct gap with both the CBM and VBM at $\Gamma$.

Figure 3 clearly demonstrates that the direct/indirect nature of the band gap is the result of the competition of the energies of several near-band-edge states. With an increase in tensile strain, the energy of the $\mathrm{CB}$ at $K 1(0,0.3,0)$, labeled as state $\mathrm{D}$ in Fig. 3(g), reduces rapidly and becomes equal to that of state $\mathrm{C}(\mathrm{CB}$ at $\Gamma$, i.e., the original $\mathrm{CBM})$ when $\varepsilon_{x}=+8 \%$. With the strain higher than $+8 \%$, state $\mathrm{D}$ has a lower energy than $\mathrm{C}$; thus, D becomes the CBM. Similar behavior occurs with the VB. The energy of state $\mathrm{B}$ at $K 1(0,0.3,0)$ increases faster than $\mathrm{A}(\mathrm{VB}$ at $\Gamma$, i.e., the original $\mathrm{VBM})$. At $\varepsilon_{x}=$ $+12 \%, \mathrm{~B}$ has a higher energy than $\mathrm{A}$ and thus represents the VBM.

On the side of compression, the CBM is always located at $\Gamma$ (state $\mathrm{C}$ ); however, the VBM demonstrates an interesting transition. First, at $\varepsilon_{x}=-2 \%$, the VB at $K 2(0.15,0,0)$, labeled as state $\mathrm{E}$ in Fig. 3(c), shows an equal energy with $\mathrm{A}$ (i.e., VB at $\Gamma$ ). With increased compression, the energy of state $\mathrm{E}$ is higher than that of $\mathrm{A}$, indicating a direct-to-indirect gap transition. At $\varepsilon_{x}=-12 \%$, the energy of a sub-VB (the pink band) state $\mathrm{F}$ at $\Gamma$ has a higher energy than $\mathrm{E}$ and becomes the VBM, showing a direct band gap at $\Gamma$.

Figure 4 shows the effect of strain $\varepsilon_{y}$ (applied in the armchair direction) on the band structure of phosphorene. It demonstrates that both expansion and compression can convert the band gap to indirect. For instance, at $\varepsilon_{y}=+8 \%$ and $+12 \%$, the gap is indirect with the VBM at $\Gamma$, while the CBM shifted from $\Gamma$ to $X$. At the side of negative strain, the gap is indirect at $\varepsilon_{y}=-12 \%$ with the VBM at $K 1(0,0.3,0)$. It is clear that the two VB states A and B compete for the VBM, and the two $\mathrm{CB}$ states $\mathrm{C}$ and $\mathrm{G}(\mathrm{CB}$ at $X)$ exchange dominance for the CBM. The energy shift of states $B$ and $G$ with strain are more prominent than their competing states $\mathrm{A}$ and $\mathrm{C}$, respectively.

\section{Strain effect on the band gap of phosphorene}

The band gap is defined as the energy difference between the CBM and VBM. From Figs. 3 and 4, the gap strongly depends on strain. In Fig. 5, the gap is plotted as a function of both strains $\varepsilon_{x}$ and $\varepsilon_{y}$.

For the strain applied along the zigzag direction in Fig. 5(a), the band gap is initially increased with tensile strain from a value of $0.91 \mathrm{eV}$ for the relaxed structure and reaches the maximal value of $0.99 \mathrm{eV}$ at $+4 \%$ strain, then drops rapidly with further increased expansion. At $\varepsilon_{x}=+12 \%$, the band gap is reduced to $0.22 \mathrm{eV}$. To see if the gap reduces to zero with further increased tensile strain, we explored even larger strain $+13 \%$ up to $+16 \%$ with a $1 \%$ increment. The gap was not found to close. It reaches a minimal value of $0.06 \mathrm{eV}$ at $+14 \%$ strain and then opens up again for larger strain.

On the side of negative strain $\varepsilon_{x}$, the gap reduces, mainly resulting from the downward shift of the CBM (see Fig. 3). At $\varepsilon_{x}=-12 \%$, the gap sharply drops to $0.04 \mathrm{eV}$ from the value of $0.55 \mathrm{eV}$ at $-10 \%$ strain. This is due to the fact that the VBM was replaced by a newly raised sub-VB (state F). To explore if the band gap reduces to zero with a larger compression, we explored the $-13 \%$ strain and found that the DFT gap indeed closed up.

In Fig. 5(a), five strain zones were identified based on their distinct band structure. Zone I is for a direct band gap within the strain range $-12 \%$ to $-10.2 \%$, in which the CBM is represented by state $\mathrm{C}$ and the VBM is given by state $\mathrm{F}$. Zone II corresponds to an indirect band gap from $-10.2 \%$ to $-2 \%$, where the VBM is state E. Zone III is a direct gap at $\Gamma$ from $-2 \%$ to $+8 \%$. Zone IV is an indirect gap from $+8 \%$ to $+11.3 \%$, where the CBM is at $(0,0.3,0)$. Zone $\mathrm{V}$ shows a direct band gap with both the CBM and VBM at $(0,0.3,0)$. The critical strains of $-10.2 \%,-2 \%,+8 \%$, and $+11.3 \%$ are the zone boundaries that are determined in the next section.

Figure 5(b) presents the band gap as a function of strain applied in the armchair direction. The reduced gap value with 
(a)

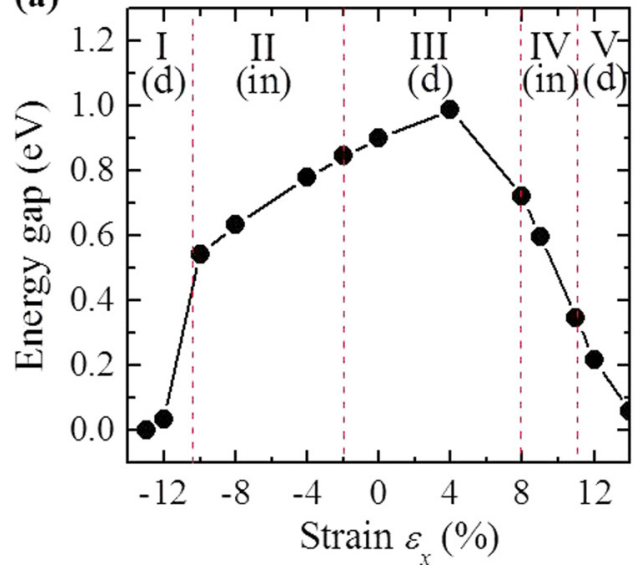

(b)

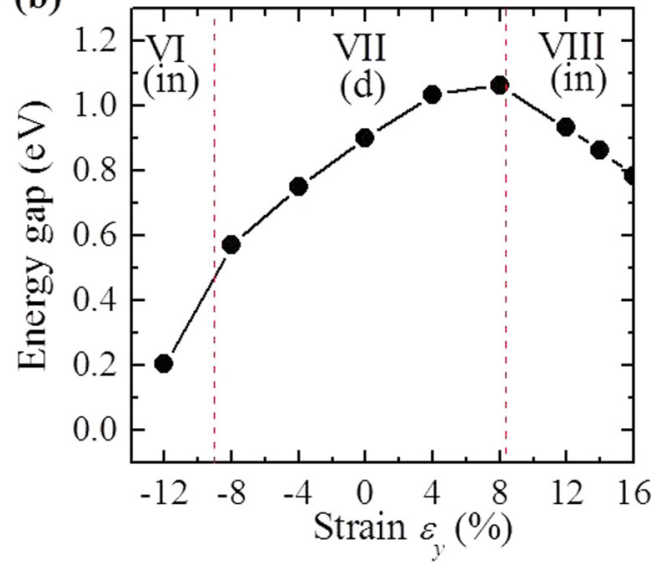

FIG. 5. (Color online) The band gap of 2D phosphorene as a function of strain $\varepsilon_{x}$ applied in the zigzag (left) and $\varepsilon_{y}$ in the armchair (right) directions, respectively. Five (three) strain zones were identified for $\varepsilon_{x}\left(\varepsilon_{y}\right)$ based on its distinct band structures. Zones I, II, III, IV, and V in (a) are corresponding to the direct (d), indirect (in), direct, indirect, and direct gap, respectively. The critical strain for the gap transition are $-10.2 \%,-2 \%,+8 \%$, and $+11.3 \%$. The gap closes up at $\varepsilon_{x}=-13 \%$. Zones VI, VII, and VIII in (b) present the indirect, direct, and indirect gap, respectively; the critical strains of the zone borders are $\varepsilon_{y}=-9 \%$ and $+6.8 \%$.

compression mainly results from the downward shift of the CBM and upward change of the VBM (see Fig. 4). The drop of the gap value at $+12 \%$ results from the fact that the CBM is replaced by the $\mathrm{CB}$ at $\mathrm{X}$ (state $\mathrm{G}$ ). Three unique strain zones are characterized. Zone VI is for an indirect band gap from $-12 \%$ to $-9 \%$ where the VBM is located at $(0,0.3,0)$. Zone VII is for direct band gap at $\Gamma$ from $-9 \%$ to $+6.8 \%$. Zone VIII shows an indirect band gap in the strain range $+6.8 \%$ to $+12 \%$, in which the CBM is at $X$.

\section{Validation of the strain effects using hybrid functionals}

Since DFT at the GGA level is known to underestimate the band gap of semiconductors, it is important to test the robustness of the calculated strain effects on the band structures of phosphorene. We used the advanced hybrid functional
HSE06 to calculate the band structures of phosphorene under different values of axial strain in the zigzag direction, and the results are presented in Fig. 6. Comparing the HSE06 predicted band structures in Figs. 6(a)-(e) with that of the DFT in Fig. 3, one can conclude that both methods give the consistent results of the strain effects on the band structures. For example, the direct band gap of the material without strain $(0 \%)$ converts to indirect under $+9 \%$ strain and then becomes direct again with $+12 \%$ strain. On the side of compression, the gap is indirect and direct under $-8 \%$ and $-12 \%$ strains, respectively. We also used the HSE06 method to calculate the band structures of phosphorene under different values of strain in the armchair direction and found that they are in great agreement with those obtained from the standard DFT.

The band gaps predicted by the DFT and HSE06 as a function of strains $\varepsilon_{x}$ and $\varepsilon_{y}$ are presented in Figs. 6(f) and
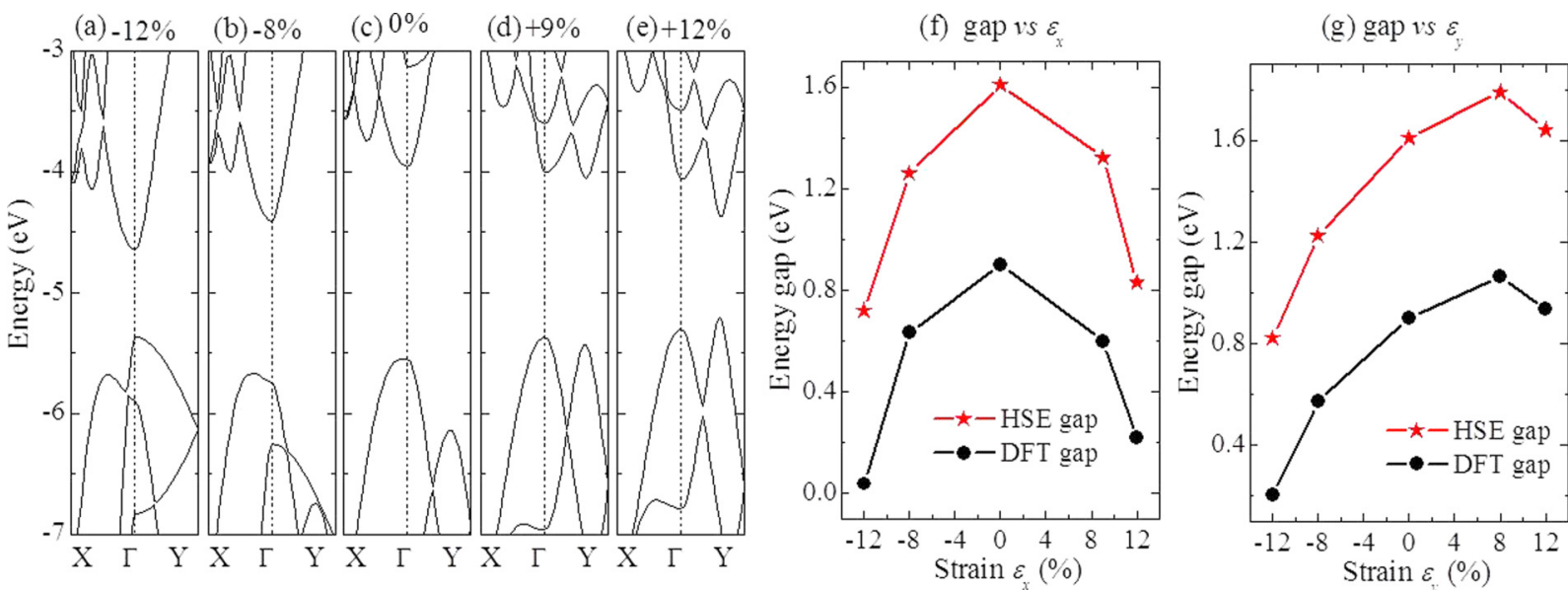

FIG. 6. (Color online) (a)-(e) Hybrid functional HSE06 predicted band structures of 2D phosphorene with different values of strain $\varepsilon_{x}$ in the zigzag direction. Starting with the relaxed structure, the band gap experiences a direct-indirect-direct transition with both tensile and compressive strain, which is in great agreement with that predicted by the standard DFT. (f), (g) Band gaps predicted by the standard DFT and HSE06 for both strains $\varepsilon_{x}$ and $\varepsilon_{y}$. Although DFT underestimates the band gap, its calculated gap-strain variation trend is consistent with that of HSE. 
$6(\mathrm{~g})$. It is clear that the HSE06 has a better prediction on the band gap value. The HSE06 calculated band gap for the relaxed phosphorene is $1.61 \mathrm{eV}$, which is $0.7 \mathrm{eV}$ higher than the DFT gap. However, both methods of the DFT and HSE06 calculated the same gap variation trends with strain. Therefore, we can conclude that DFT correctly predicts the general trends of strain effects on the band structure and near-gap states in phosphorene, including the direct-indirect band gap transition and the gap variation trends with strain.

\section{E. Strain effect on the near-band-edge orbitals}

To determine the critical strain in which the direct-indirect band gap transition occurs, we plotted the energies of the near-band-edge states A-G (labeled in Fig. 3 and Fig. 4) as a function of strain in Fig. 7. As seen from Figs. 3 and 4, these are the states that compete for the CBM and VBM, thus their energies determine the direct/indirect nature of the gap. In Fig. 7, the energies of the states display a nearly linear function with strain. For instance, the energy of state B increases with a positive strain $\varepsilon_{x}$ while decreasing with a negative strain $\varepsilon_{x}$. In contrast, states $\mathrm{D}$ and $\mathrm{F}$ show opposite trends. The energy of state E remains unaffected by strain. This linear shift of energy is not unique to phosphorene. It is also observed in other semiconducting nanostructures [28,29,31-38,51-54].

In Fig. 7, the conduction states were represented by hollowdashed lines, while valence states are given by solid lines. In Fig. 7(a), state C represents the CBM from $-12 \%$ strain up to $+8 \%$, at which the energy of state $\mathrm{D}$ equals to that of $\mathrm{C}$. From $+8 \%$ to $+12 \%$ strain, $\mathrm{D}$ has a lower energy than $\mathrm{C}$, thus $\mathrm{D}$ represents the CBM. The energy crossover of these two states gives the critical strain $+8 \%$ for the direct-indirect band gap transition.

For the VBs, two states A and B compete for the VBM at the positive strain, while three states $\mathrm{A}, \mathrm{E}$, and $\mathrm{F}$ battle at compression. State A represents the VBM for positive strain up to $+11.3 \%$, where $C$ catches up and becomes the VBM. For the negative strain, the energy of $\mathrm{A}$ was first exceeded by $\mathrm{E}$ at $-2 \%$ strain, and $\mathrm{E}$ becomes the $\mathrm{VBM}$ in the strain range from $-2 \%$ to $-10.2 \%$. At $-10.2 \%$ strain, $\mathrm{F}$ catches up and represents the VBM for compression higher than $-10.2 \%$.

In the case of strain $\varepsilon_{y}$ in Fig. 7(b), the energy crossover of two conduction states $\mathrm{C}$ and $\mathrm{G}$ occurs at $+6.8 \%$ strain and that of two valence states $\mathrm{A}$ and $\mathrm{B}$ at $-9 \%$.

To understand the different trends of states A-G in Fig. 7, their electronic orbitals, including charge distribution and wave function, were explored. The wave function character was examined by projecting the wave function onto $s, p$, and $d$ orbitals at each ionic site. It was found that states $\mathrm{A}$ and $\mathrm{E}$ are $p_{z}$ orbitals, $\mathrm{B}$ is $p_{y}, \mathrm{C}$ is dominated by $p_{z}(86 \%)$ mixed with $p_{y}, \mathrm{D}$ is dominated by $p_{y}(56 \%)$ with a mixture of $s$ orbital, $\mathrm{F}$ is $p_{x}$, and $\mathrm{G}$ is dominated by $p_{x}(59 \%)$ mixed with $s$ orbital. Their electron density contour plots and wave function character are presented in Fig. 8.

We developed a general mechanism [29] to explain the different energy shifts with strain based on the HeitlerLondon's exchange energy model [55]. The different energy shifts with strain were found to be closely related to the bonding/antibonding nature of the orbitals [29]. In the Heitler-London model, the energies of the bonding and antibonding states are given by the equations,

$$
\begin{gathered}
E_{\text {bonding }}=2 E_{0}+\frac{e^{2}}{R}+\frac{K+H}{1+S^{2}} \\
E_{\text {antibonding }}=2 E_{0}+\frac{e^{2}}{R}+\frac{K-H}{1-S^{2}},
\end{gathered}
$$

where $E_{0}$ is the energy for an isolated atom, $K$ represents the classical Coulomb energy between the electron-electron and electron-ion interactions, and $S$ is the overlap integral of the orbitals between different atomic sites, which is usually much smaller than 1. The square of $S$ is even smaller. Therefore, the exchange integral term $H$ may be playing a dominant role in determining the different energy variation behaviors with strain in the bonding and antibonding situation. The exchange $H$ is given by

$$
\begin{aligned}
H= & \iint \psi_{a}^{*}\left(r_{1}\right) \psi_{b}^{*}\left(r_{2}\right)\left(\frac{1}{r_{12}}-\frac{1}{r_{2 a}}-\frac{1}{r_{1 b}}\right) \\
& \times \psi_{b}\left(r_{1}\right) \psi_{a}\left(r_{2}\right) d r_{1} d r_{2},
\end{aligned}
$$

where the exchange $H$ is contributed from either nonclassical electron-electron (i.e., $\frac{1}{r_{12}}$, positive) or electron-ion interaction (i.e., $-\frac{1}{r_{2 a}}-\frac{1}{r_{1 b}}$, negative). For $s$ orbitals or any mixed orbitals in which electron density are not extremely localized, the contribution of the electron-ion interaction is dominated over the electron-electron interaction in the exchange $H$. As the atomic distance increases (corresponding to a positive tensile strain), the energy contributed from the electron-ion interaction increases more rapidly compared to the energy reduction of the electron-electron contribution [see Eq. (3)], which results in an increased value for $H$. An increased $H$ value also causes the bonding energy $E_{\text {bonding }}$ to increase and the antibonding energy $E_{\text {antibonding }}$ to decrease, based in Eqs. (1) and (2). These trends are represented by the schematic in Fig. 8(h). For the case of nonbonding, in which the wave function overlap is minimal, the energy is expected to be insensitive to strain.

Now to examine the electron density contour plots and wave function character in Fig. 8 to determine their bond nature. State A in Fig. 8(a) suggests nonbonding in the $x$ and an antibonding character in the $y$ direction based on its sign of phase factor along the $y$ direction. B is bonding in the $x$ while displaying antibonding in the $y$ direction. $\mathrm{C}$, which is dominated by $p_{z}$ orbital, illustrates a bonding nature in the $x$ while nonbonding in the $y$ direction. States D, E, and F demonstrate antibonding, nonbonding, and antibonding, respectively, in the $x$ axis. State $\mathrm{G}$ is antibonding in the $y$ direction. State $\mathrm{D}$ is a mixture of $p_{y}$ and $s$ orbitals. Since the overlap of the $p_{y}$ orbital in the $x$ direction is small, the $s$ orbital is plotted in Fig. 8(d) to determine its bonding/antibonding status in the $x$ direction. The same case is applied to state $\mathrm{G}$ in Fig. $8(\mathrm{~g})$.

The bond nature of these seven states combined with the schematic in Fig. 8(h) can be used to explain their energy variation in Fig. 7. For example, D is antibonding in the $x$ direction and is expected to decrease with tensile strain from Fig. 8(h). This is in agreement with the curve of D in Fig. 7(a). $\mathrm{B}$ is bonding in the $x$ while antibonding in the $y$ direction. According to Fig. 8(h), its energy is expected to increase with $\varepsilon_{x}$ while decreasing with $\varepsilon_{y}$, which is consistent with Fig. 7. Other curves in Fig. 7 can be explained in the same manner. 

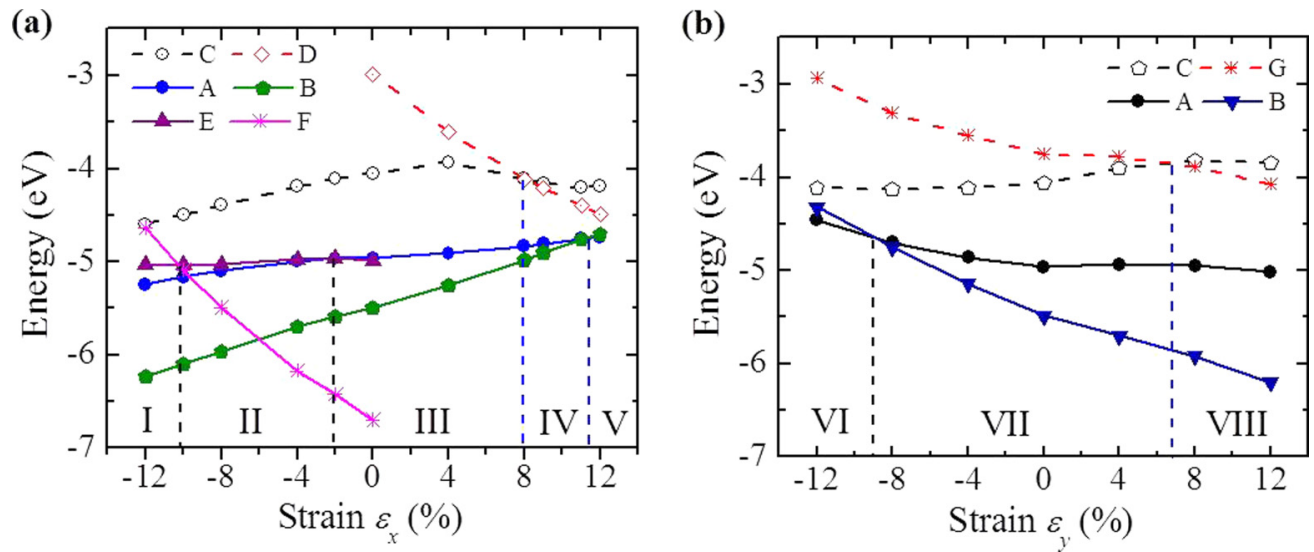

FIG. 7. (Color online) The energies of the near-edge states A-G as a function of strain applied in the zigzag (left) and armchair (right) direction, respectively. The critical strains for the direct-indirect gap transition are determined by the energy crossover of competing states. (a) Energy crossover of the competing VB states A, E, and F occurs at $\varepsilon_{x}=-2 \%,-10.2 \%$, and $+11.3 \%$ strains. The energies of the competing $\mathrm{CB}$ states $\mathrm{C}$ and $\mathrm{D}$ are equal at $\varepsilon_{x}=+8 \%$ strain. (b) Energies of the VB states $\mathrm{A}$ and $\mathrm{B}$ crosses at $\varepsilon_{y}=-9 \%$, and two $\mathrm{CB}$ states $\mathrm{C}$ and $\mathrm{G}$ meets at $\varepsilon_{y}=+6.8 \%$. Strain Zones I-VIII are also labeled.

\section{F. Strain effect on the effective masses of charge carriers}

The effective masses of the electron and hole can be readily calculated according to the formula $m^{*}=\hbar^{2}\left(\frac{d^{2} E}{d k^{2}}\right)^{-1}$ from the band structure. For relaxed phosphorene, the effective mass of the electron is predicted to be $1.24 m_{e}$ in the zigzag and $0.16 m_{e}$ in the armchair direction. The effective mass of the hole is $4.92 m_{e}$ in the zigzag and $0.15 m_{e}$ in the armchair direction. These calculated effective masses are in agreement with other theoretical work [26,27]. The significantly smaller effective masses in the armchair direction suggest that charge carriers prefer transport in that direction.

The effective masses of the electron and hole as a function of both strains $\varepsilon_{x}$ and $\varepsilon_{y}$ are presented in Fig. 9. The effective masses of the electron and hole can be dramatically tuned by strain. In addition, it was found that the sudden jump (drop) in the effective masses occurs around the strain zone boundaries (i.e., critical strains) for the direct-indirect band (a) State $A$

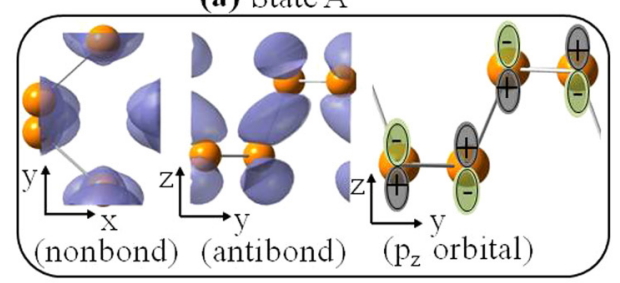

(b) State B
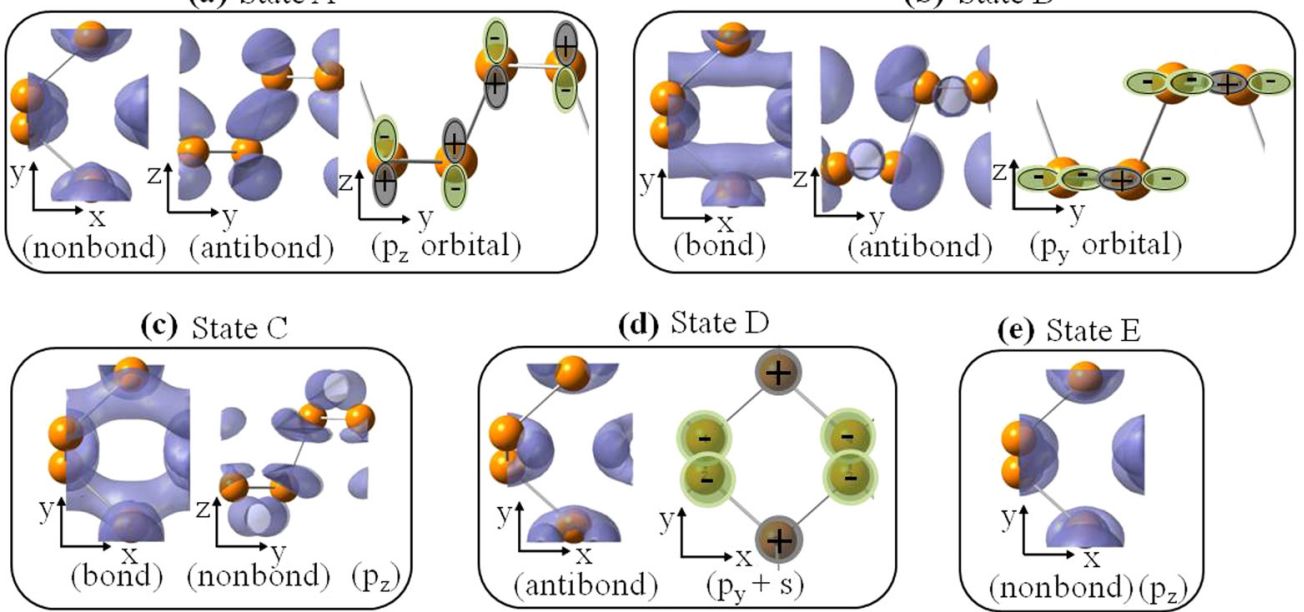

(f) State F

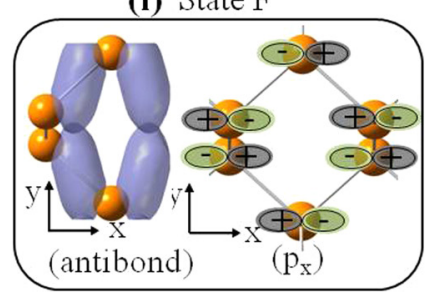

(d) State D

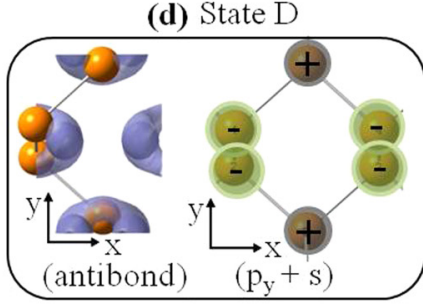

(g) State G

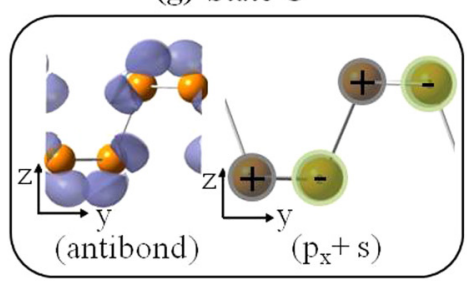

(e) State E

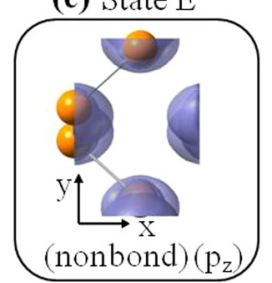

(h) schematic

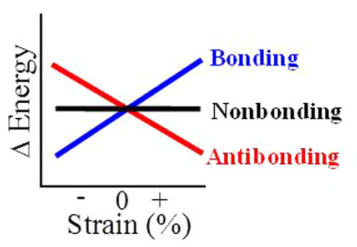

FIG. 8. (Color online) (a)-(g) The electron density contour plots and schematic of the wave function character (i.e., the projected major orbital and sign of phase factor) of the near-band-edge states A-G in 2D phosphorene. Their dominant orbitals and bond status (in the horizontal axis) were listed at the bottom of each state. (h) A schematic of energy response to axial strain for three typical cases of bonding, nonbonding, and antibonding. 

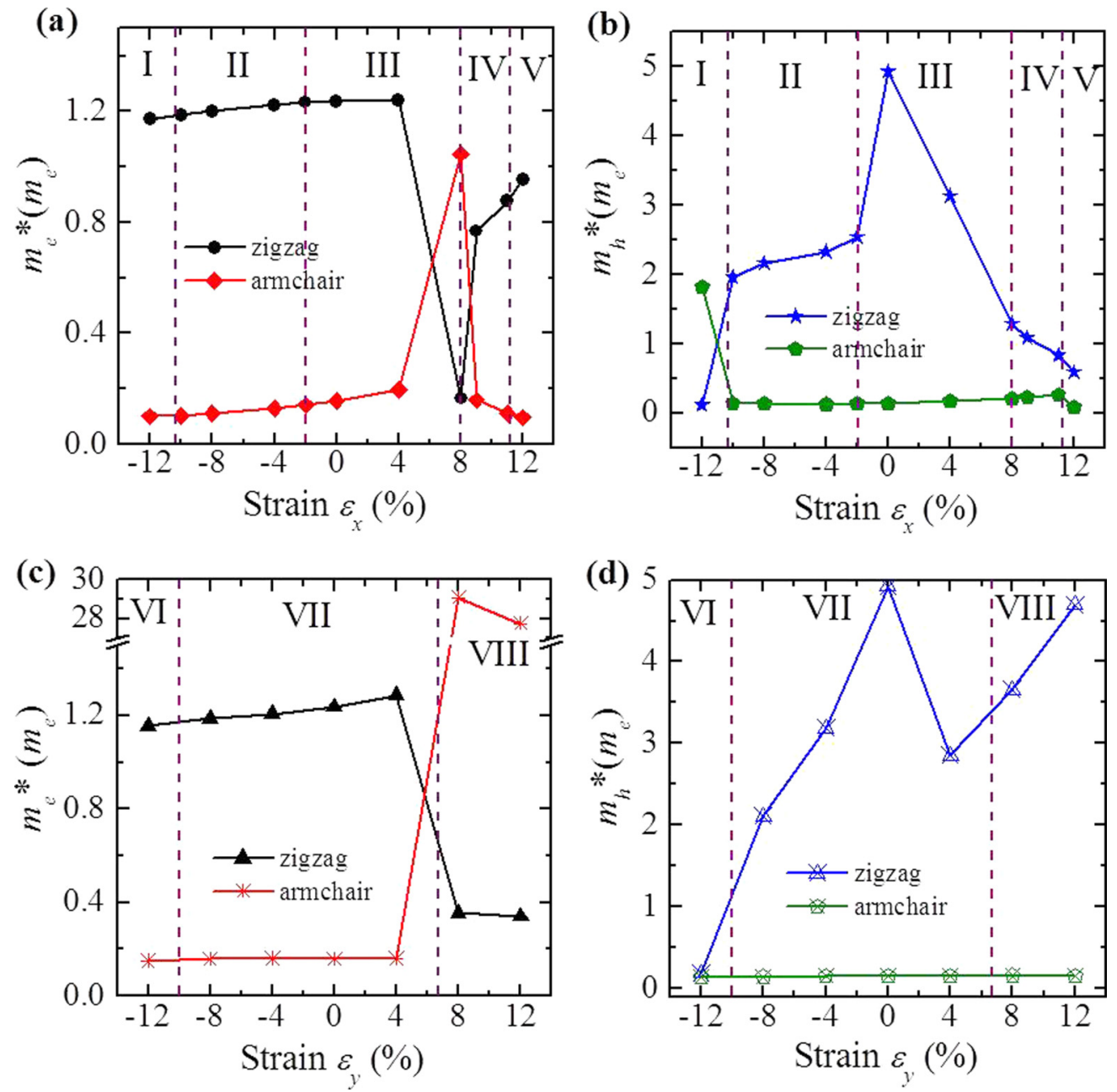

FIG. 9. (Color online) Effective masses of the electron (left) and hole (right) as a function of strain $\varepsilon_{x}$ (top) and $\varepsilon_{y}$ (bottom). Five (three) strain zones for $\varepsilon_{x}\left(\varepsilon_{y}\right)$ are also labeled. The sharp shift in effective masses occurs around the zone boundaries for the direct-indirect gap transition.

gap transition. For example, in Fig. 9(a), the effective mass of the electron in the zigzag direction is an order of magnitude bigger than that along the armchair direction in Zones I, II, and III. At the zone boundary of III and IV, the effective mass in the zigzag direction drops sharply, while in the armchair direction it jumps suddenly. This sharp transition suggests the zigzag direction is favored for electron transport [26]. Figure 9(b) shows that the hole prefers transport in the armchair direction in Zones II-V; however, in Zone I, it favors the zigzag direction.

For the case of strain applied in the $y$ direction, Fig. 9(c) suggests that the armchair direction is favored for the electron transport in Zones VI and VII, while the situation becomes opposite in Zone VIII. For the hole in Fig. 9(d), the effective mass in the armchair direction is insensitive to strain, while in the zigzag direction it experiences a dramatic shift. The much smaller value of effective mass in the armchair direction indicates the hole predominately prefers transport in the armchair direction in Zone VII and VIII. However, the sharp reduction of the effective mass in the zigzag direction at Zone VI makes this axis competitive with the armchair direction for the hole transport.
The effective mass presented in Fig. 9 is a direct consequence of the strain effect on the band structure in Figs. 3 and 4. In particular, the sharp change in the effective masses results from the direct-indirect band gap transition. For example, to understand the sudden shift of the effective mass at $\varepsilon_{x}=$ $+8 \%$ in Fig. 9(a), refer to the band structure of Fig. 3(h) along $\Gamma$ to $Y$. Compared to the $+4 \%$ strain in Fig. $3(\mathrm{~g})$, the downward shift of state $\mathrm{D}$ at $+8 \%$ strain largely reduces the band dispersion at state $\mathrm{C}$, thus increasing the effective mass of the electron dramatically at $\varepsilon_{x}=+8 \%$. When $\varepsilon_{x}$ is bigger than $+8 \%$, the CBM is shifted away from $\mathrm{C}$ to $\mathrm{D}$; thus, the effective mass of the electron was calculated from $\mathrm{D}$, which has a much smaller effective mass resulting from the more dispersive band structure. Another sharp shift in the effective mass of the hole occurs at $\varepsilon_{x}=-12 \%$ in Fig. 9(b), which is a direct consequence of Fig. 3(a). At $\varepsilon_{x}=-12 \%$, the energy of sub VB state F exceeds $E$ and becomes the VBM. The effective mass along the $x$ direction is now calculated based on this new state F instead of E. Similarly for the case of $\varepsilon_{y}$ strain, the striking transition of the effective mass of the electron from $+4 \%$ to $+8 \%$ strain in Fig. 9 (c) results from the $+6.8 \%$ critical strain. 


\section{CONCLUSIONS}

Using $a b$ initio calculations, we provided a detailed analysis of strain effects on the electronic band structure of 2D phosphorene. We found the following to be true. (i) Phosphorene can withstand a tensile stress and strain up to $10 \mathrm{~N} / \mathrm{m}$ and $30 \%$, respectively. (ii) The band gap of $2 \mathrm{D}$ phosphorene has direct-indirect-direct transitions with axial strain. (iii) Five strain zones with distinct electronic band structure were identified, and the critical strains for the zone boundaries were determined. (iv) Although the DFT method is known to underestimate the band gap of semiconductors, it was proven to correctly predict the strain effect on the electronic properties with verification via the hybrid functional method. (v) The origin of the gap transition was revealed, and a general mechanism was developed to explain the near-band-edge energy shifts according to the bond nature of their electronic orbitals. (vi) In relaxed phosphorene, effective masses of the electron and hole in the armchair direction are an order of magnitude smaller than that of the zigzag direction, suggesting that the armchair direction is favored for carrier transport. (vii) Effective masses can be dramatically tuned by strain. (viii) The sharp jump/drop in the effective masses occurs at the zone boundaries of direct-indirect gap transition.
Phosphorene has demonstrated superior mechanical flexibility and can hold a large tensile strain of $30 \%$, which opens doors for applications in flexible displays. Having a direct band gap is essential for materials in optical applications. Our paper shows that a moderate $-2 \%$ strain in the zigzag direction can trigger the direct-to-indirect band gap transition. Our predicted strain Zones II, IV, VI, and VIII should be avoided for optical applications due to the indirect band gap in the material within these strain zones. Carrier mobility is an essential parameter to determine performance of electronics and it is inversely dependent on the effective masses. We demonstrated that strain can dramatically tune the effective masses; thus, it can be used to modify carrier mobility of phosphorene in electronics applications.

\section{ACKNOWLEDGMENTS}

This work is supported by the Faculty Research Initiative Fund from School of Letters and Sciences at Arizona State University (ASU) to Peng. The authors thank ASU Advanced Computing Center for providing resources. Drs. F. Tang and L. Yang are acknowledged for the helpful discussions. Dr. Tang is also greatly appreciated for the critical review of the manuscript.
[1] K. S. Novoselov, A. K. Geim, S. V. Morozov, D. Jiang, M. I. Katsnelson, I. V. Grigorieva, S. V. Dubonos, and A. A. Firsov, Nature 438, 197 (2005).

[2] Y. B. Zhang, Y. W. Tan, H. L. Stormer, and P. Kim, Nature 438, 201 (2005).

[3] B. Radisavljevic, A. Radenovic, J. Brivio, V. Giacometti, and A. Kis, Nat Nanotechnol 6, 147 (2011).

[4] L. Li, Y. Yu, G. Jun Ye, Q. Ge, X. Ou, H. Wu, D. Feng, X. Hui Chen, and Y. Zhang, Nat Nanotechnol 9, 372 (2014).

[5] H. Liu, A. T. Neal, Z. Zhu, Z. Luo, X. Xu, D. Tománek, and P. D. Ye, Acs Nano 8, 4033 (2014).

[6] E. S. Reich, Nature 506, 19 (2014).

[7] F. Xia, H. Wang, and Y. Jia, Nat. Commun. 5, 4458 (2014).

[8] A. S. Rodin, A. Carvalho, and A. H. Castro Neto, Phys. Rev. Lett. 112, 176801 (2014).

[9] Y. Takao and A. Morita, Physica B \& C 105, 93 (1981).

[10] V. Tran, R. Soklaski, Y. Liang, and L. Yang, Phys. Rev. B 89, 235319 (2014).

[11] R. S. Jacobsen, K. N. Andersen, P. I. Borel, J. Fage-Pedersen, L. H. Frandsen, O. Hansen, M. Kristensen, A. V. Lavrinenko, G. Moulin, H. Ou, C. Peucheret, B. Zsigri, and A. Bjarklev, Nature 441, 199 (2006).

[12] M. R. Falvo, G. J. Clary, R. M. Taylor, V. Chi, F. P. Brooks, S. Washburn, and R. Superfine, Nature 389, 582 (1997).

[13] Y. Q. Cai, A. H. Zhang, Y. P. Feng, C. Zhang, H. F. Teoh, and G. W. Ho, J. Chem. Phys. 131, 224701 (2009).

[14] Y. D. Wang, N. Lu, H. B. Xu, G. Shi, M. J. Xu, X. W. Lin, H. B. Li, W. T. Wang, D. P. Qi, Y. Q. Lu, and L. F. Chi, Nano Res. 3, 520 (2010).

[15] A. Thean and J. P. Leburton, Appl. Phys. Lett. 79, 1030 (2001).

[16] X. L. Wu and F. S. Xue, Appl. Phys. Lett. 84, 2808 (2004).
[17] L. Seravalli, M. Minelli, P. Frigeri, P. Allegri, V. Avanzini, and S. Franchi, Appl. Phys. Lett. 82, 2341 (2003).

[18] S. Mazzucato, D. Nardin, M. Capizzi, A. Polimeni, A. Frova, L. Seravalli, and S. Franchi, Mater. Sci. Eng.: C 25, 830 (2005).

[19] Y. G. Wang, Q. L. Zhang, T. H. Wang, W. Han, and S. X. Zhou, J. Phys. D 44, 125301 (2011).

[20] A. I. Hochbaum and P. Yang, Chem. Rev. 110, 527 (2009).

[21] X. H. Peng and P. Logan, Appl. Phys. Lett. 96, 143119 (2010).

[22] K. S. Kim, Y. Zhao, H. Jang, S. Y. Lee, J. M. Kim, K. S. Kim, J.-H. Ahn, P. Kim, J.-Y. Choi, and B. H. Hong, Nature 457, 706 (2009).

[23] C. Lee, X. D. Wei, J. W. Kysar, and J. Hone, Science 321, 385 (2008).

[24] A. Castellanos-Gomez, M. Poot, G. A. Steele, H. S. J. van der Zant, N. Agrait, and G. Rubio-Bollinger, Nanoscale Research Letters 7, 1 (2012).

[25] Q. Wei and X. Peng, Appl. Phys. Lett. 104, 251915 (2014).

[26] R. Fei and L. Yang, Nano Lett. 14, 2884 (2014).

[27] J. Qiao, X. Kong, Z.-X. Hu, F. Yang, and W. Ji, Nat. Commun. 5, 4475 (2014).

[28] A. Copple, N. Ralston, and X. Peng, Appl. Phys. Lett. 100, 193108 (2012).

[29] X. Peng and A. Copple, Phys. Rev. B 87, 115308 (2013).

[30] G. Signorello, E. Lörtscher, P. A. Khomyakov, S. Karg, D. L. Dheeraj, B. Gotsmann, H. Weman, and H. Riel, Nat. Commun. 5, 3655 (2014).

[31] X. H. Peng, A. Alizadeh, N. Bhate, K. K. Varanasi, S. K. Kumar, and S. K. Nayak, J. Phys.: Condens. Matter 19, 266212 (2007).

[32] X.-H. Peng, S. Ganti, A. Alizadeh, P. Sharma, S. K. Kumar, and S. K. Nayak, Phys. Rev. B 74, 035339 (2006). 
[33] S. Sreekala, X. H. Peng, P. M. Ajayan, and S. K. Nayak, Phys. Rev. B 77, 155434 (2008).

[34] P. Logan and X. H. Peng, Phys. Rev. B 80, 115322 (2009).

[35] X. H. Peng, A. Alizadeh, S. K. Kumar, and S. K. Nayak, Int. J. of Applied Mechanics 1, 483 (2009).

[36] X. H. Peng and S. Velasquez, Appl. Phys. Lett. 98, 023112 (2011).

[37] X.-H. Peng, F. Tang, and P. Logan, J. Phys.: Condens. Matter 23, 115502 (2011).

[38] X. H. Peng, F. Tang, and A. Copple, J. Phys.: Condens. Matter 24, 075501 (2012).

[39] W. Kohn and L. J. Sham, Phys. Rev. 140, A1133 (1965).

[40] J. P. Perdew, K. Burke, and M. Ernzerhof, Phys. Rev. Lett. 77, 3865 (1996).

[41] P. E. Blochl, Phys. Rev. B 50, 17953 (1994).

[42] G. Kresse and D. Joubert, Phys. Rev. B 59, 1758 (1999).

[43] J. Heyd, G. E. Scuseria, and M. Ernzerhof, The J. Chem. Phys. 118, 8207 (2003).
[44] J. Heyd, G. E. Scuseria, and M. Ernzerhof, The J. Chem. Phys. 124, 219906 (2006).

[45] G. Kresse and J. Furthmuller, Phys. Rev. B 54, 11169 (1996).

[46] G. Kresse and J. Furthmuller, Comput. Mater. Sci. 6, 15 (1996).

[47] A. Brown and S. Rundqvist, Acta Cryst. 19, 684 (1965).

[48] J. B. Hudson, Surface Science (John Wiley \& Sons, Inc., New York, 1998).

[49] D. Roundy and M. L. Cohen, Phys. Rev. B 64, 212103 (2001).

[50] W. Luo, D. Roundy, M. L. Cohen, and J. W. Morris, Phys. Rev. B 66, 094110 (2002).

[51] M. Zhou, Z. Liu, Z. F. Wang, Z. Q. Bai, Y. P. Feng, M. G. Lagally, and F. Liu, Phys. Rev. Lett. 111, 246801 (2013).

[52] S. Huang and L. Yang, Appl. Phys. Lett. 98, 093114 (2011).

[53] K.-H. Hong, J.-K. Yoon, and P.-R. Cha, Appl. Surf. Sci. 253, 2776 (2006).

[54] K.-H. Hong, J. Kim, S.-H. Lee and J. K. Shin, Nano Lett 8, 1335 (2008).

[55] R. M. White, Quantum Theory of Magnetism, 3rd ed. (Springer, New York, 2006). 